\title{
Mott transition in a cavity-boson system: A quantitative comparison between theory and experiment
}

\author{
Rui Lin ${ }^{1}$, Christoph Georges ${ }^{2}$, Jens Klinder ${ }^{2}$, Paolo Molignini ${ }^{3}$, Miriam Büttner ${ }^{4}$, \\ Axel U. J. Lode ${ }^{4}$, R. Chitra ${ }^{1}$, Andreas Hemmerich ${ }^{2,5}$, Hans Keßler ${ }^{2 *}$ \\ 1 Institute for Theoretical Physics, ETH Zürich, 8093 Zurich, Switzerland \\ 2 Zentrum für Optische Quantentechnologien and Institut für Laser-Physik, \\ Universität Hamburg, 22761 Hamburg, Germany \\ 3 Cavendish Laboratory, 19 JJ Thomson Avenue, Cambridge CB3 0HE, United Kingdom \\ 4 Institute of Physics, Albert-Ludwig University of Freiburg, \\ Hermann-Herder-Straße 3, 79104 Freiburg, Germany \\ 5 The Hamburg Center for Ultrafast Imaging, \\ Luruper Chaussee 149, 22761 Hamburg, Germany \\ * hkessler@physnet.uni-hamburg.de
}

April 28, 2021

\begin{abstract}
The competition between short-range and cavity-mediated infinite-range interactions in a cavity-boson system leads to the existence of a superfluid phase and a Mott-insulator phase within the self-organized regime. We quantitatively compare the steady-state phase boundaries of this transition measured in experiments and simulated using the Multiconfigurational Time-Dependent Hartree Method for Indistinguishable Particles. To make the problem computationally viable, we represent the full system by the exact many-body wave function of a two-dimensional four-well potential. We argue that the validity of this representation comes from the nature of both the cavity-atomic system and the Bose-Hubbard physics, and verify that it only induces small systematic errors. The experimentally measured and theoretically predicted phase boundaries agree reasonably. We thus propose a new approach for the quantiative numerical determination of the superfluid-Mott-insulator phase boundary.
\end{abstract}

\section{Contents}

\begin{tabular}{lll}
\hline 1 & Introduction & 2
\end{tabular}

\begin{tabular}{|lll}
2 & Experimental setup and measurement protocol & 4
\end{tabular}

2.1 Cavity-BEC system and superfluid-Mott-insulator transition 4

\begin{tabular}{lll}
\hline 2.2 & Measurement protocol & 6
\end{tabular}

\begin{tabular}{llr}
\hline & Simulation Methodology & 8
\end{tabular}

3.1 Numerical method 8

$3.2 \quad$ Reduction of system dimensionality and rescaling of the contact interaction strength

3.3 Four-well model 
5 Conclusions and Discussion 13

\begin{tabular}{|lr|}
\hline A Summary of methods and parameters & 14
\end{tabular}

B Experimental phase diagram with fast ramping protocol 14

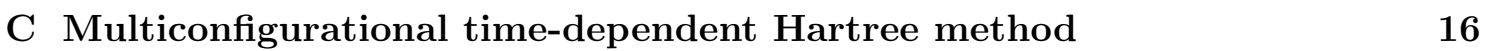

D Calculation of the effective two-dimensional atomic contact interaction strength
E Effects induced by the confining potential and the size of the reduced $\begin{array}{ll}\text { lattice } & 17\end{array}$

\begin{tabular}{lr}
\hline References & 18
\end{tabular}

\section{Introduction}

During the past decade, experimental and theoretical progress using quantum gases to realize models of solid state physics has made it possible to study many-body effects in isolated and highly controllable scenarios $[1-3]$. In particular, the interplay between light and matter creates a unique platform for the exploration of a multitude of exotic behaviors in quantum systems 4 -15. Compared to traditional solid state systems, light-matter systems provide an opportunity for more complex and flexible setup construction and finetuning of system parameters. The achieved knowledge can then be applied to much more complex and integrated systems as, for example, in solid state physics and material science.

One topic receiving enduring interest is the many-body effects in ultracold atomic systems, particularly the coherence between particles in the superfluid phase and its loss in the Mott-insulator phase of a lattice system. The transition between these two phases was first realized by controlling an optical lattice potential in cold-atom systems in three [16 and two dimensions [17,18, respectively. A similar transition can also be realized through the coupling to an optical cavity as sketched in Fig. 1. Driven by an external laser and with cavity-mediated interactions, effectively two-dimensional Bose-Einstein condensates (BECs) can self-organize into a superlattice [7]. As the drive increases, a transition between a self-organized superfluid (SSF) phase and a self-organized Mott-insulator (SMI) phase is observed experimentally 15, 19 and investigated theoretically 20 24. This transition stems from a combination of the short-range interaction due to $s$-wave scattering between the atoms and the infinite-range interaction mediated by the cavity. The cavity-BEC system can thus reproduce a quantum-optical version of the Bose-Hubbard model.

Hitherto, a direct quantitative comparison between experiment and theory on the SSFSMI transition has not been presented because of the enormous computational effort required, although it is crucial for future applications. For example, machine learning techniques have recently been applied onto various physical systems [25 29], including ultracold atomic systems $30-32$. However, it requires a mass amount of data which is far from the capability of experimental measurement. In contrary, quantitative numerical simulations are suitable for this task due to its more precise tunability of system parameters and its shorter time scale in data collection. 
(a)

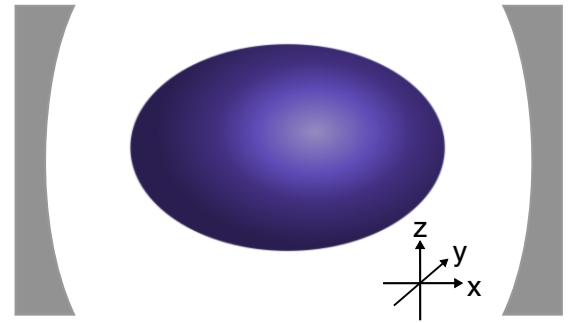

(b)

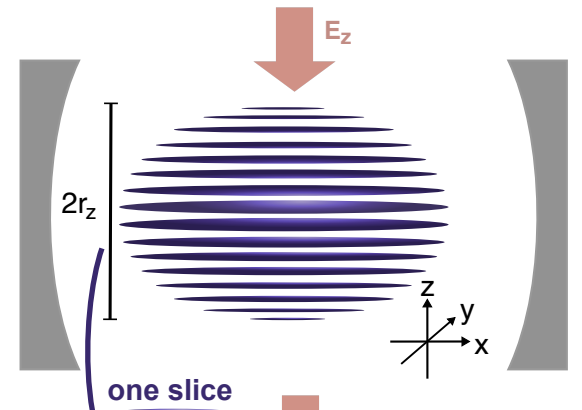

(c)

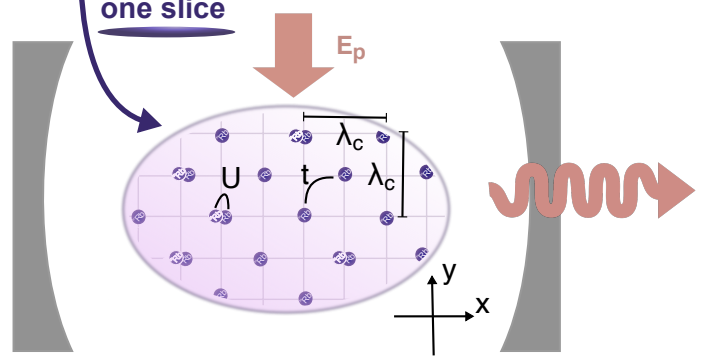

Figure 1: Sketch of the experimental system. The atoms are first prepared as (a) a threedimensional BEC, and then cut into (b) two-dimensional slices by an external laser pump along $z$ direction. (c) Due to the pumping laser along $y$ direction and the interplay with the cavity, they finally self-organize into a checkerboard lattice with wavelength $\lambda_{c}$ along both $x$ and $y$ directions. The onset of the self-organization can be detected by leaking photons. After the checkerboard superlattice is formed, the system can be mapped to a Bose-Hubbard model with tunneling strenght $t$ and on-site interaction $U$.

In this work, we perform such a quantitative comparison, in particular of the phase diagram, by employing the Multiconfigurational Time-Dependent Hartree Method for Indistinguishable Particles (MCTDH-X) [33 39]. This method captures the many-body effects beyond the Gross-Pitaevskii mean-field limit, including but not limited to the coherence between the atoms. In order to keep the computational complexity within a tractable range, we construct a simplification scheme for the simulations by exploiting the nature of the cavity-BEC system and the superfluid-Mott-insulator transition. This simplification scheme nevertheless retains the many-body essence of the system to a satisfactory degree, and quantitatively reproduces the phase boundary in agreement with the experiments in a wide parameter range. The comparison is summarized in the phase diagram in Fig. 2.

This work is organized as follows. In Sec. 2, we introduce the system as well as the experimental setup, and we describe our experimental protocol for obtaining the experimental phase diagram. In Sec. 3, we first briefly introduce MCTDH-X, and then propose a simplification scheme, which is well adapted and specialized to the cavity-BEC system and MCTDH-X. This simplification scheme essentially approximates the full system by a two-dimensional four-well model. In Sec. 4, we compare the experimental and simulation results, and discuss the origin of the discrepancy between them. Finally, we draw conclusions in Sec. 5 . 


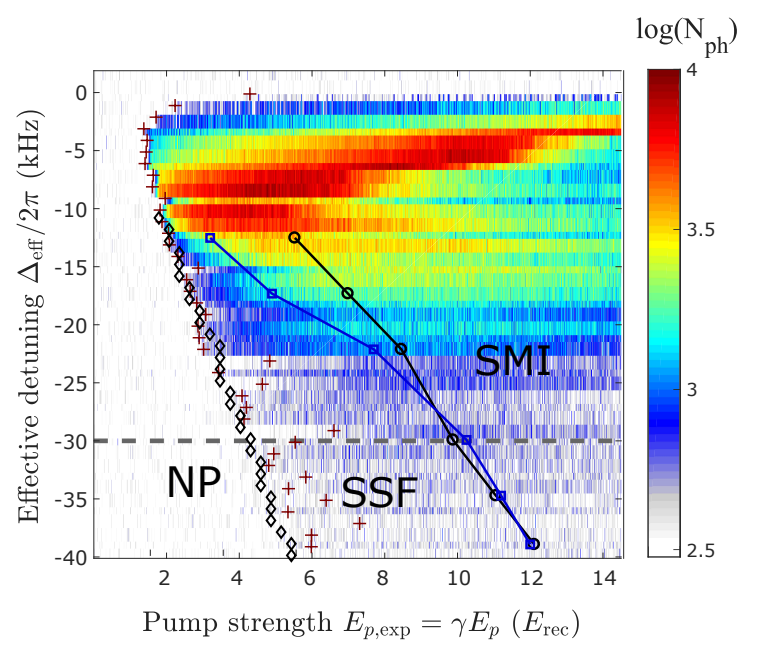

Figure 2: The steady-state phase diagram identifying the normal BEC phase (NP), the self-organized superfluid phase (SSF) and the self-organized Mott-insulator phase (SMI). It is plotted against effective cavity-pump detuning $\Delta_{\text {eff }}$ and pump strength $E_{p \text {,exp }}=$ $\gamma E_{p}$ in units of the recoil energy $E_{\text {rec }}$, where $\gamma=1.36$ is a calibration factor between the pump strength used in experiments and simulations. To determine the experimental NP-SSF boundary (dark red crosses), we use the slow ramping protocol with ramping time $T_{r}=40 \mathrm{~ms}$ and measure the photon number leaking through one of the cavity mirrors (background color). The boundary is then defined by a rapid increase in the photon number. It is compared to the simulated NP-SSF boundary (black diamonds). To determine the experimental SSF-SMI boundary (black circles), we use the fast ramping protocol with $T_{r}=20 \mathrm{~ms}$ and measure the momentum space density. The boundary is then defined by a rapid increase in the central peak width. It is compared to the simulated SSF-SMI boundary (blue squares) which is obtained through our proposed simplification scheme. The simplification scheme induces systematic errors in the predicted boundary of roughly $\pm 0.5 E_{\text {rec }}$. The black and blue lines are guide to the eyes, and the gray dashed line marks the detuning $\Delta_{\text {eff }}$ used in Figs. 4 and 5.

\section{Experimental setup and measurement protocol}

\subsection{Cavity-BEC system and superfluid-Mott-insulator transition}

The experimental system, as sketched in Fig. 1, consists of a laser-driven Bose-Einstein condensate (BEC) of $N_{3 \mathrm{D}}=55,000$ Rubidium-87 $\left({ }^{87} \mathrm{Rb}\right)$ atoms dispersively coupled to a high-finesse optical cavity with strength $\Omega_{g}$. The atoms are magnetically trapped in a three-dimensional harmonic potential with trapping frequencies $\left(\omega_{x}, \omega_{y}, \omega_{z}\right)=2 \pi \times$ $(25.2,202.2,215.6) \mathrm{Hz}$. In the absence of external drive, the ensemble forms a ThomasFermi cloud with measured radii $\left(r_{x}, r_{y}, r_{z}\right)=(26.8,3.3,3.1) \mu \mathrm{m}$ [Fig. 11(a)]. The threedimensional atomic cloud is overlapped with the fundamental mode of the high-finesse optical cavity oriented along the $x$ direction. The cavity resonance frequency $\omega_{c}$ and wave vector $k_{c}$ correspond to a wavelength of $\lambda_{c}=803 \mathrm{~nm}$ and a recoil energy of $E_{\mathrm{rec}}=$ $\hbar^{2} k_{c}^{2} / 2 m_{\mathrm{Rb}}=\hbar \times 2 \pi \times 3.55 \mathrm{kHz}$. The cavity has a relatively low field decay rate of $\kappa=2 \pi \times 4.45 \mathrm{kHz}$, and operates in the sub-recoil regime [40]. The atomic cloud is then loaded into an external optical lattice oriented along the $z$ direction, which is given by $E_{\text {ext }}(z)=E_{z} \cos ^{2}\left(2 \pi z / \lambda_{z}\right)$ with wavelength $\lambda_{z}=803 \mathrm{~nm}$ and depth $E_{z}=12.5 E_{\mathrm{rec}}$. The strong external lattice suppresses tunneling along the $z$ direction and renders the system 
into effective two-dimensional slices spanned on the $x-y$ plane, as illustrated in Fig. 1(b).

After preparing and loading the BEC into $E_{\text {ext }}(z)$, the ensemble is transversely pumped along the $y$ direction by a laser with effective pump strength $E_{p}$ and frequency $\omega_{p}=2 \pi \lambda_{p} / c$, which forms an effective standing-wave optical lattice $E_{y}(y)=E_{p} \cos ^{2}\left(2 \pi y / \lambda_{p}\right)$. We work in the dispersive regime $\omega_{p} \gg \omega_{a}$ using pump light at a wavelength of $\lambda_{p}=803 \mathrm{~nm}$. This is far detuned from the relevant atomic transition of ${ }^{87} \mathrm{Rb}$ at $\lambda_{a}=795 \mathrm{~nm}$. We note that the atoms and the cavity are both red-detuned from the pump light $\Delta_{a}=\omega_{a}-\omega_{p}<0$, $\Delta_{c}=\omega_{c}-\omega_{p}<0$.

Combining all the aforementioned components of the setup, we can write down the full many-body Hamiltonian of the cavity-BEC system [7, 41, 42,

$$
\begin{aligned}
\hat{\mathcal{H}}= & \int d x d y d z \hat{\Psi}^{\dagger}\left(\frac{\mathbf{p}^{2}}{2 m_{\mathrm{Rb}}}+V_{\mathrm{trap}}+V_{\mathrm{opt}}\right) \hat{\Psi}+\frac{g_{3 \mathrm{D}}}{2} \int d x d y d z \hat{\Psi}^{\dagger} \hat{\Psi}^{\dagger} \hat{\Psi} \hat{\Psi} \\
V_{\mathrm{trap}}= & \frac{m_{\mathrm{Rb}}}{2}\left(\omega_{x}^{2} x^{2}+\omega_{y}^{2} y^{2}+\omega_{z}^{2} z^{2}\right) \\
V_{\mathrm{opt}}= & -E_{p} \cos ^{2}\left(k_{c} y\right)-E_{z} \cos ^{2}\left(k_{c} z\right) \\
& +\hbar U_{0}|\alpha|^{2} \cos ^{2}\left(k_{c} x\right)+\sqrt{\hbar E_{p}\left|U_{0}\right|}\left(\alpha+\alpha^{*}\right) \cos \left(k_{c} x\right) \cos \left(k_{c} y\right) .
\end{aligned}
$$

Here, $\hat{\Psi} \equiv \hat{\Psi}(x, y, z)$ is the atomic annihilation operator, $m_{\mathrm{Rb}}=1.44 \times 10^{-25} \mathrm{~kg}$ is the mass of the ${ }^{87} \mathrm{Rb}$ atoms, $g_{3 \mathrm{D}}$ is the atom-atom contact interaction strength, and $U_{0}=\Omega_{g}^{2} / \Delta_{a}=$ $-2 \pi \times 0.36 \mathrm{~Hz}$ is the single-atom light shift. The cavity field, pumping laser, and external lattice are near resonance $\lambda_{c} \approx \lambda_{p} \approx \lambda_{z}$. A summary of the experimental parameters is given in Appendix A.

The cavity field is treated as coherent light and represented by its expectation value $\alpha$. It follows the equations of motion $7,41,42$

$$
\begin{aligned}
\partial_{t} \alpha & =\left[i\left(\Delta_{c}-N_{3 \mathrm{D}} U_{0} B\right)-\kappa\right] \alpha-i \sqrt{\frac{E_{p}\left|U_{0}\right|}{\hbar}} N_{3 \mathrm{D}} \theta \\
\theta & =\int d x d y d z \rho(x, y, z) \cos \left(k_{c} x\right) \cos \left(k_{c} y\right) \\
B & =\int d x d y d z \rho(x, y, z) \cos ^{2}\left(k_{c} x\right),
\end{aligned}
$$

where $\rho(x, y, z)=\left\langle\hat{\Psi}^{\dagger} \hat{\Psi}\right\rangle / N_{3 \mathrm{D}}$ is the spatial density distribution. Under this treatment, the cavity field effectively imposes a one-body potential upon the atoms, as evident in the second line of Eq. (1c). This treatment of the cavity field is legitimate as long as the cavity fluctuations $\left\langle\delta a^{2}\right\rangle=\left\langle a^{\dagger} a\right\rangle-|\langle a\rangle|^{2}$ are small, which is indeed the case except near the self-organization boundary [23, 43, 45], which will be introduced in detail below.

The atomic many-body wave function of the steady state of the cavity-BEC system can be obtained by solving Eqs. (1) and (2) self-consistently. At large pump strength, the atoms reduce the potential energy by self-organizing into a checkerboard lattice with lattice spacing $\lambda_{c}$ along the $x$ and $y$ directions as depicted in Fig. 1(c), and constructively scatter photons from the pump into the cavity $7,9,42,44$. In a steady state, the dominant part $\left[\cos \left(k_{c} x\right) \cos \left(k_{c} y\right)\right]$ of the cavity-induced potential has an effective depth

$$
E_{\mathrm{cb}}=\left|\frac{2 E_{p} U_{0} N_{3 \mathrm{D}} \theta\left(\Delta_{c}-N_{3 \mathrm{D}} U_{0} B\right)}{\left(\Delta_{c}-N_{3 \mathrm{D}} U_{0} B\right)^{2}+\kappa^{2}}\right| .
$$

This self-organization transition can be mapped to the Hepp-Lieb normal-superradiant phase transition of the Dicke model [46 [49], and is accompanied by the spontaneous breaking of the $\mathbb{Z}_{2}$ symmetry, which is reflected by the sign of $\theta$. A positive (negative) $\theta$ corresponds to an even (odd) lattice configuration. 
Deep in the self-organized phase, the atoms progressively localize on the checkerboard lattice sites as the pump strength increases and the induced optical potential deepens. Coherence between atoms at different lattice sites gradually decays, leading to a second transition from the SSF phase to the SMI phase [15, 23, 24]. During this transition, cavity fluctuations are indeed minimal $23,43,45]$, validating our mean-field treatment of the cavity field. The SSF and SMI phases behave similar to the superfluid and Mott-insulator phases, respectively, of the usual Bose-Hubbard model

$$
H_{\mathrm{BH}}=-t \sum_{\langle i, j\rangle}\left(b_{i}^{\dagger} b_{j}+b_{j}^{\dagger} b_{i}\right)+\frac{U}{2} \sum_{i} b_{i}^{\dagger} b_{i}^{\dagger} b_{i} b_{i},
$$

where $b_{i}$ is the annihilation operator for bosonic atoms at the $i$-th lattice site, $t$ is the tunneling strength, $U$ is the on-site interaction, and $\langle i, j\rangle$ indicates the summation is over nearest neighbors. In this model, a superfluid is characterized by a fluctuating particle number per site and phase coherence of the whole ensemble due to large tunneling between different lattice sites. On the contrary, in a Mott-insulator, phase coherence is lost, the particle fluctuations vanish and the number of atoms per lattice site is fixed due to the suppressed tunneling. The differences between the two phases lead to distinct behaviors in various quantities, including the variance of on-site atom number $\operatorname{Var}=\left\langle\left(b_{i}^{\dagger} b_{i}\right)^{2}\right\rangle-\left\langle b_{i}^{\dagger} b_{i}\right\rangle^{2}, 50,51$ and the momentum space density distribution $15,18,22,24,52,56$

$$
\tilde{\rho}(\mathbf{k})=\left\langle\hat{\Psi}^{\dagger}(\mathbf{k}) \hat{\Psi}(\mathbf{k})\right\rangle .
$$

Since the former quantity is hard to measure experimentally, we choose $\tilde{\rho}(\mathbf{k})$ as our main quantity of interest for defining the phase boundary. As the system enters the Mottinsulator phase from the superfluid phase, a significant increase in the full width at half maximum (FWHM) $\mathcal{W}$ of the central peak in the momentum space density distribution can be observed [15 18, 23, 24,52,54] accompanying the loss of phase coherence. The transition between the two phases is thus smooth and has only weak criticality. For a $d$-dimensional system, it is in the same universality class as a $(d+1)$-dimensional $X Y$ model [57].

In the cavity-BEC system, the total number of atoms enters the equation of motion Eq. (2) and effectively modifies the cavity detuning. Meanwhile, the number of atoms per site, equivalent to the filling factor in the Bose-Hubbard model, is an important ingredient in determining the SSF-SMI boundary [17,52,58,60]. Therefore, a quantitative comparison between experiment and theory necessitates an estimate to the number of atoms in each two-dimensional slice as well as at each lattice site near the center of the harmonic trap. For simplicity, we assume a uniform distribution of the atoms in the central cuboid of the three-dimensional harmonic trap, such that $x / r_{x}, y / r_{y}$ and $z / r_{z}$ are all within the interval $[-1 / 2,1 / 2]$. In this region, since the two-dimensional slices are $\lambda_{c} / 2$ apart from each other along the $z$ direction, there are $2 r_{z} / \lambda_{z} \approx 8$ slices in total and each slice contains roughly $N_{2 \mathrm{D}} \approx 6,900$ atoms. Once the system enters the self-organized phase, the atoms in each slice will further form a superlattice with two lattice sites per area of $\lambda_{c}^{2}$. There are thus $2 r_{x} r_{y} / \lambda_{c}^{2}=275$ lattice sites in the considered rectangle on each slice, and each of the lattices contains $\nu \approx 25$ atoms.

\subsection{Measurement protocol}

The comparison between the experimental and simulated phase diagrams involves both the NP-SSF and the SSF-SMI boundaries for the steady state. In experiments, we fix the effective detunings

$$
\Delta_{\mathrm{eff}}=\Delta_{c}-\frac{1}{2} N_{3 \mathrm{D}} U_{0}
$$


while ramping up the pump strength linearly from zero to $E_{p, \exp }=14.5 E_{\text {rec }}$ within a time $T_{r}$. There is a trade-off when choosing an appropriate ramping time, and we choose two different ramping times for the measurement of different observable to best approximate the steady-state phase boundaries.

In the vicinity of the NP-SSF boundary, the photonic behavior is dominating in the system due to significant cavity fluctuations. As the cavity decay rate is relatively small in comparison to the effective detuning $\kappa<\left|\Delta_{\text {eff }}\right|$, the cavity field experiences a retardation effect when crossing the steady-state NP-SSF boundary [9]. As a result, the dynamical NPSSF boundary depends on the ramping time, and converges to the steady-state boundary with long $T_{r}$. With a ramping time of $T_{r}=40 \mathrm{~ms}$, the hysteresis area is negligibly small and the steady-state boundary can be well approximated [9]. During the linear ramping of the pump strength, we record along the way the intracavity photon number $N_{\mathrm{ph}}$ leaking through one of the cavity mirrors using a single photon counting module (SPCM). This is plotted in logarithmic scale in Fig. 2, We then subtract the dark count offset from the measured signal and determine the phase boundary according to the threshold of the measured photon number $N_{\mathrm{ph}} \approx 300$. The measured NP-SSF boundary will later be compared to the simulated one as a calibration.

On the other hand, deep inside the self-organized phase, the cavity fluctuations vanish and atomic behavior becomes dominant. In this region, atom loss becomes a key factor. A decrease in the atom number effectively increases $\left|\Delta_{\text {eff }}\right|$ when both $\Delta_{c}$ and $U_{0}$ are negative [cf. Eq. [6] ], and it generally indicates that a higher pump strength $E_{p}$ is required to achieve the same lattice depth [cf. Eq. (3)]. Therefore, all phase boundaries are shifted towards higher pump strength when atom loss occurs. Since a longer ramping time implies a larger atom loss and hence a larger shift in the boundary, a fast ramp with $T_{r}=20 \mathrm{~ms}$ is thus preferred for the measurement of the steady-state SSF-SMI boundary. This boundary is extracted from the momentum space density distribution $\tilde{\rho}(\mathbf{k})$ [15, 16, 18, 21, 23. . To measure the momentum distribution we repeat the experiment several times where we stop at a certain pump strength for ballistic expansion of the sample. After switching off all the trapping potentials and a $25 \mathrm{~ms}$ long time of flight, we detect the momentum distribution using single-shot absorption images. Thereafter, we can extract the width of the central peak from the distribution, and mark the SSF-SMI boundary at the pump strength where the width starts to increase. The measured SSF-SMI boundary is marked as the black line with circles in the phase diagram Fig. 2. We note that with the fast ramping protocol $T_{r}=20 \mathrm{~ms}$, the measured dynamical NP-SSF is indeed significantly shifted towards larger pump strengths when compared to the slow ramping protocol (see Appendix B).

Caution needs to be taken when analyzing the experimental measurements. The experimentally calibrated pump strength $E_{p \text { exp }}$ is different from the pump strength $E_{p}$ entering the Hamiltonian. In experiments, the pump laser is not strictly monochromatic. On the one hand, the pump strength is calibrated by measuring the energy difference between the first and the third Bloch band at zero quasi-momentum. This is done by an active modulation of the lattice depth and measuring the resonance frequency for parametric heating of the BEC 61, 62]. Such measurement considers effects from electromagnetic waves of all frequencies. On the other hand, only the component with frequency $\omega_{p}$ can scatter into the cavity and contribute to the effective cavity-induced potential of the atoms and thus the self-organization. As a result, the effective pump strength $E_{p}$ entering Eqs. (1) and (2) is different from the experimental one $E_{p \text {,exp }}$ obtained directly through calibration in experiments. These two pump strengths are related through a calibration factor

$$
\gamma \equiv E_{p, \exp } / E_{p}>1
$$

which is not measurable through experiments without applying significant hardware changes 
to the system. To determine the factor $\gamma$, we need to compare the experimental and simulated phase diagrams, and require that they coincide with each other. This comparison will be performed in Sec. 3.2 after obtaining the simulated NP-SSF boundary.

\section{Simulation Methodology}

\subsection{Numerical method}

We use the approach Multiconfigurational Time-Dependent Hartree Method for Indistinguishable Particles (MCTDH-X) to simulate the steady state of the system and extract the observables of interest [33 39], like the momentum space density distribution and the cavity field expectation value. MCTDH-X is able to solve problems beyond the Gross-Pitaevskii mean-field limit, and capture the correlations between atoms as well as quantum fluctuations in the many-body states. The method relies on a variational ansatz for the manybody state, which is a symmetrized product of multiple optimized functions, or orbitals. The number of orbitals $M$ controls the simulation accuracy. Ideally, the exact solution of the numerical problem is found when an infinite number of orbitals is used [35, 36, 63]. MCTDH-X has been successfully applied for investigating the static and dynamic behaviors of Bose-Hubbard systems 22, 23, 64, 65]. A more detailed description of the method can be found in Appendix C.

The number of orbitals used in a simulation depends on the nature of the quantum state of interest. For example, the formation of the cavity-induced potential and thereby the self-organization of the atoms can well be observed in the mean-field limit with $M=1$ orbital [23, 34, 66]. In contrast, to correctly describe a Mott-insulator state, the number of orbitals should be at least as large as the number of lattice sites [22, 23, 39, 63, 67]. Since the required computational resources scale as $\left(\begin{array}{c}N-M+1 \\ M\end{array}\right)$ 35], given the currently available processors, it is computationally unfeasible to simulate with MCTDH-X the full experimental cavity-BEC system, which consists of 55,000 atoms and thousands of lattice sites in three dimensions. Therefore, we need to simplify the problem and reduce the number of orbitals and particles needed for the numerical model. We will now elaborate on the methodology for choosing this simplification.

\subsection{Reduction of system dimensionality and rescaling of the contact interaction strength}

The computational complexity can be significantly reduced by lowering the system dimensionality. We argue that the system can be well represented by a two-dimensional model, and determine the effective atom-atom interaction strength in this model.

In experiments, the system is divided into two-dimensional slices by the deep external optical lattice. The hopping between two slices is vanishingly small, and thus the slices are independent from each other on the atomic level [cf. Eq. (1)]. On the other hand, atoms from all slices collectively contribute to the cavity field, and therefore they are strongly coupled to each other through the cavity [cf. Eq. (2)]. In order to represent the full system by one two-dimensional slice, we propose to decouple the slices by simulating Eqs. (1) and (2) with $N_{2 \mathrm{D}}$ atoms at $z=0$, and using the scaling of parameters 42

$$
U_{0} \mapsto \tilde{U}_{0}=U_{0} \frac{N_{3 \mathrm{D}}}{N_{2 \mathrm{D}}}, \quad \alpha \mapsto \alpha \sqrt{\frac{N_{2 \mathrm{D}}}{N_{3 \mathrm{D}}}} .
$$

Under this scaling, the equations of motion for the cavity field [Eq. (2)] as well as the cavity-induced potential $V_{\text {opt }}$ [Eq. (1)] remain invariant for a fixed atomic density profile. 
We thus expect that the atomic many-body wave function of the two-dimensional system obtained from Eq. (8) approximately reproduces the wave function of the original system at $z=0$,

$$
\left|\Psi_{2 \mathrm{D}}(x, y)\right\rangle \approx\left|\Psi_{3 \mathrm{D}}(x, y, z=0)\right\rangle .
$$

This approach requires a knowledge of the effective contact interaction in the twodimensional slice. This parameter is crucial to the formation of Mott insulation. The strength $g_{2 \mathrm{D}}$ is estimated according to the harmonic trapping frequencies and the corresponding Thomas-Fermi radii [68],

$$
N_{2 \mathrm{D}} g_{2 \mathrm{D}}=\frac{\pi m_{\mathrm{Rb}}}{4} \frac{r_{x}^{4} \omega_{x}^{3}}{\omega_{y}}
$$

which yields $g_{2 \mathrm{D}} \approx 0.34 \hbar^{2} / m_{\mathrm{Rb}}$ for $N_{2 \mathrm{D}}=6,900$, as explained in detail in Appendix $\mathrm{D}$.

With the effective single slice, we can simulate the physics of the realistic experimental system using MCTDH-X at different pump lattice depths $E_{p}$ and effective detunings $\Delta_{\text {eff }}$ in simulations, and as the first observable we choose the cavity field strength. The formation of the density wave and the accompanying macroscopic activation of the cavity field can already be captured with sufficient precision by using $M=1$ orbital in the mean-field limit. We therefore use the notation $\alpha_{\mathrm{MF}}\left(E_{p}, \Delta_{\text {eff }}\right)$ for the simulated cavity field strength. The simulated $\alpha_{\mathrm{MF}}$ can be used to determine the NP-SSF boundary, which is drawn where $\left|\alpha_{\mathrm{MF}}\right|^{2} \approx 0.1$. Although this choice of threshold is different from the experimental one, it should not induce substantial difference due to the rapid increase of photon number across the boundary. Both criteria are chosen based on the analytically expected boundary and the respective limitations in experiments and simulations. With the simulation results, the calibration factor $\gamma$ [cf. Eq. (7)] for the experimental pump strength can now be calculated to be $\gamma=1.36$. This is determined by requiring that the measured NP-SSF boundary and the simulated one, which are fitted as $\Delta_{\text {eff }} / 2 \pi=\left(-8.536 E_{p \text {,exp }} / E_{\text {rec }}+6.305\right) \mathrm{kHz}$ and $\Delta_{\text {eff }} / 2 \pi=\left(-11.616 E_{p} / E_{\text {rec }}+5.834\right) \mathrm{kHz}$ respectively, have the same slope as functions of pump strengths. The experimental and simulated NP-SSF boundaries indeed collapse upon each other when this calibration factor is taken into account (cf. Fig. 2). We emphasize that once the effective contact interaction strength $g_{2 \mathrm{D}}$ and the calibration factor $\gamma$ are determined, there is no more free parameter in the simulation when compared to the experimental system.

\subsection{Four-well model}

We now proceed to simulate the SSF-SMI transition. A proper description of this transition requires at least one orbital for each lattice site. Given the large number of atoms and lattice sites, it is impractical to simulate the quantum state of the full two-dimensional system, and thus further simplification of the model is needed. Since the SSF-SMI transition is mainly driven by the competition between on-site interaction and hopping between nearest-neighboring sites, the loss of superfluidity of the whole system should already be quantitatively captured by a local representation. A minimal choice for such a local representation is a unit cell consisting of four lattice sites in the center of the harmonic trap, which is shown in Fig. 3(b).

This four-well potential can be described by the Hamiltonian

$$
\hat{\mathcal{H}}_{4 \text { well }}=\int d x d y \hat{\Psi}^{\dagger}\left(\frac{\mathbf{p}^{2}}{2 m_{\mathrm{Rb}}}+V_{4 \mathrm{well}}\right) \hat{\Psi}+\frac{g_{2 \mathrm{D}}}{2} \int d x d y \hat{\Psi}^{\dagger} \hat{\Psi}^{\dagger} \hat{\Psi} \hat{\Psi}
$$



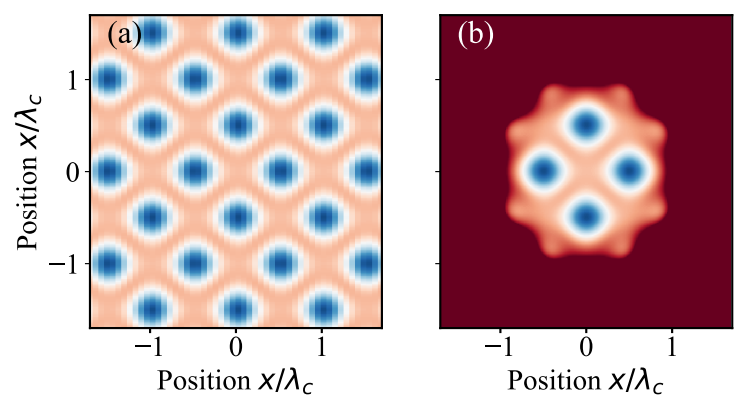

Figure 3: A comparison between (a) the cavity-induced lattice potential $V_{\text {opt }}(z=0)$ [Eq. (1)] and (b) the four-well potential $V_{4 \text { well }}$ [Eq. (12)]. Each of the four wells faithfully reproduces the lattice sites of the lattice.

where a tight non-harmonic confining potential is applied on top of the optical lattice

$$
V_{\text {well }}=\tilde{V}_{\text {opt }}+V_{\text {conf }} \text {. }
$$

The ideal confining potential should be relatively flat in the center of the system $x^{2}+y^{2}<$ $\lambda_{c}^{2}$, but form a rapidly increasing wall surrounding the four wells at $x^{2}+y^{2}>\lambda_{c}^{2}$. This can be achieved by using an algebraic function of $x^{2}+y^{2}$ with high power. For example, the following confining potential is chosen for our simulation:

$$
V_{\text {conf }}(x, y)=13 E_{\text {rec }}\left(x^{2}+y^{2}\right)^{4} / \lambda_{c}^{8} .
$$

We note that this is not the unique choice for the confining potential, and simulated SSFSMI boundary should not be sensitive to the choice (see Appendix E).

However, a straightforward implementation of the tight confining potential can easily distort the underlying optical lattice, because the equations of motion [Eqs. (1), (2)] are solved self-consistently and the solution can be very sensitive to slight changes of parameters, especially near the self-organization boundary. We thus make use of the previously simulated expectation value of the cavity field $\alpha_{\mathrm{MF}}\left(E_{p}, \Delta_{\text {eff }}\right)$ to determine the depths of the cavity-induced potential, i.e., $U_{0}\left|\alpha_{\mathrm{MF}}\right|^{2}$ and $2 \sqrt{\hbar E_{p} U_{0}} \operatorname{Re}\left(\alpha_{\mathrm{MF}}\right)$, which is equivalent to using

$$
\tilde{V}_{\text {opt }}(x, y)=V_{\text {opt }}\left(x, y, z=0, \alpha=\alpha_{\mathrm{MF}, \text { odd }}\right) .
$$

The $\mathbb{Z}_{2}$ symmetry of the cavity-BEC system corresponds to two energetically degenerate states, which are distinguishable by a $\pi$ phase shift of the intracavity field $\alpha_{\mathrm{MF} \text {, even }}=$ $-\alpha_{\mathrm{MF}, \text { odd }}$. Here we explicitly choose the one corresponding to the odd configuration, whose lattice sites are located at desired positions $\left(0, \pm \lambda_{c} / 2\right)$ and $\left( \pm \lambda_{c} / 2,0\right)$. The fourwell potential is compared to the original lattice in Fig. 3. Indeed, the shape of each of the four wells precisely recreates the shape of the each lattice site of the original optical lattice.

With four sites in total and each containing $\nu \approx 25$ atoms, we perform the simulations with $\tilde{N}=100$ atoms and $\tilde{M}=4$ orbitals subject to one-body potential $V_{4 \text { well }}$ and contact interaction with strength $g_{2 \mathrm{D}}=0.34 \hbar^{2} / m_{\mathrm{Rb}}$. In terms of the quantities related to the SSF-SMI transition, for example the momentum space density distribution and the onebody correlation function between neighboring sites, the four-well model Eq. (12) should produce the same result as the full three-dimensional experimental setup from Eqs. (1) and (2). A summary of the simulation approaches and parameters is given in Appendix A.

MCTDH-X generates a numerically highly accurate many-body wave function for the four-well system. The representation of the full effective optical lattice by our four-well 
model is a crucial non-trivial simplfication; the validity of this simplification is based on the nature of the SSF-SMI phase transition and the geometry of the system. The finite size effect of this minimal representation for the full lattice system provides the main source of systematic errors in the simplification scheme. More specifically, the transition point of a Bose-Hubbard model is subject to finite size effect, and is increased by tens of percents in terms of the ratio $t / U$ compared to the thermodynamic limit 50,51. However, in a cavity-BEC system, the ratio $t / U$ decreases exponentially as the pump strength $E_{p}$ increases [23]. As a result, the shift of the SSF-SMI boundary due to finite size effect in terms of $E_{p}$ is negligible. As a confirmation, we compare the phase boundary obtained for different numbers of lattice sites in Appendix $\mathrm{E}$. The simulated boundaries show a systematic variance of roughly $0.5 E_{\mathrm{rec}}$, and the result has indeed already converged with the four-well model.

\section{Results}
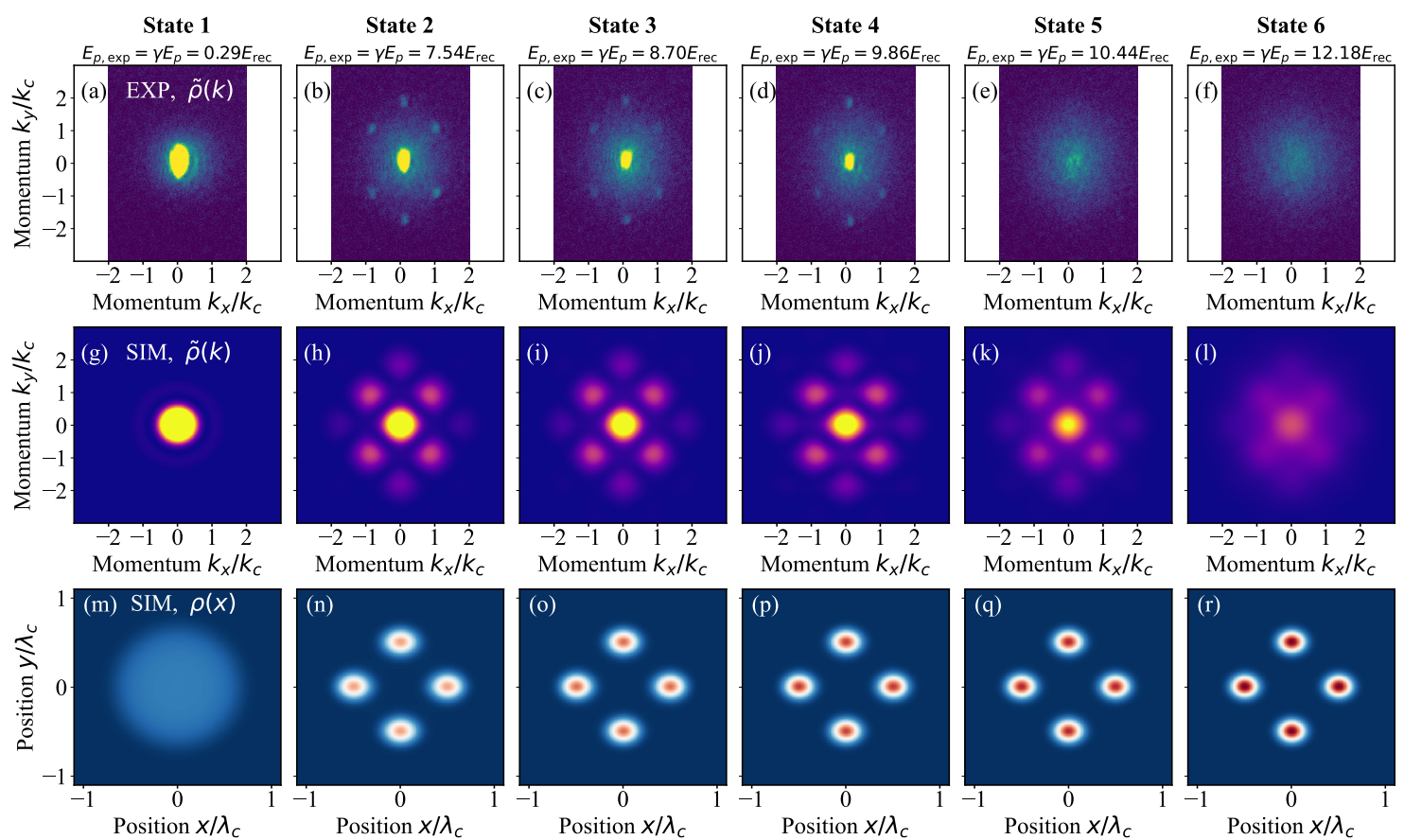

Figure 4: (a-f) Experimentally measured and (g-l) simulated momentum space density distributions $\tilde{\rho}\left(k_{x}, k_{y}\right)$, and (m-r) simulated real space density distributions $\rho(x, y)$ of six different parameter sets. The chosen states range from normal BEC state to SSF states to SMI states. They are simulated or measured at $\Delta_{\text {eff }}=-2 \pi \times 30 \mathrm{kHz}$ and different pump strengths $E_{p}$. These pump strengths correspond respectively to the points 1 to 6 as indicated in Fig. 5(b).

The momentum space density distribution $\tilde{\rho}(\mathbf{k})$ measured from experiments and calculated from simulations can be used to extract the SSF-SMI phase boundary. The obtained phase diagram of the cavity-BEC system against pump strength $E_{p}$ and effective detuning $\Delta_{\text {eff }}$ is shown in Fig. 2, It serves as a map to identify the three different phases of matter, NP, SSF, and SMI, which are realized in both experiments and simulations. To illustrate the system behavior in the three different phases, we choose a series of states at $\Delta_{\text {eff }}=-2 \pi \times 30 \mathrm{kHz}$, and show their simulated and experimentally measured density 

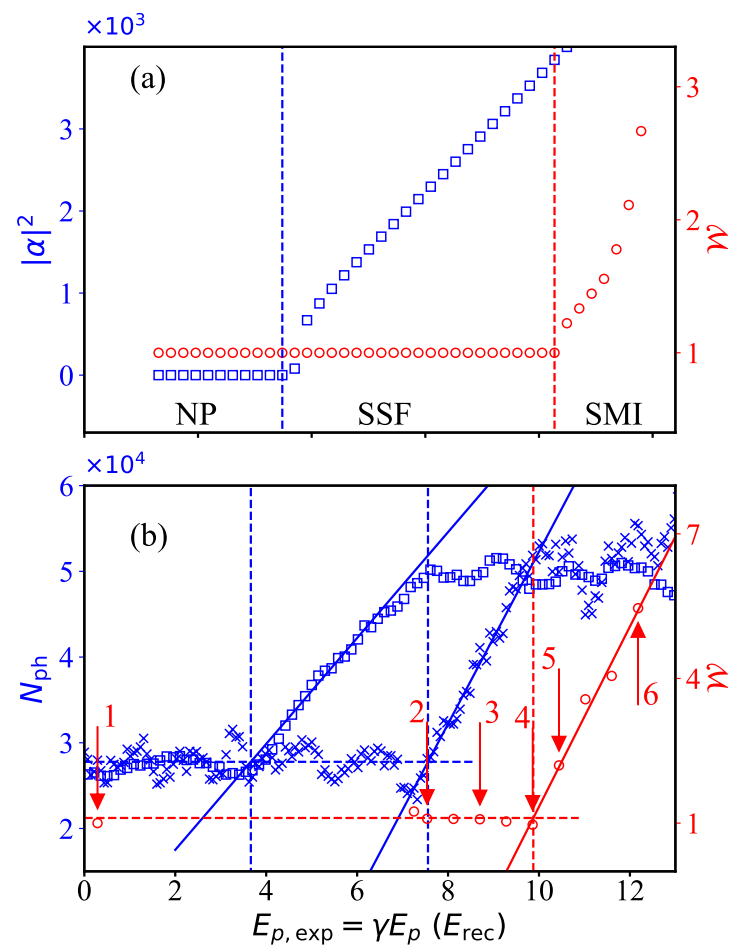

Figure 5: Cavity field magnitude $|\alpha|^{2}$, intracavity photon number $N_{\mathrm{ph}}$, and the relative width of the central Bragg peak $\mathcal{W}$ as a function of pump strength $E_{p, \exp }=\gamma E_{p}$ at fixed detuning $\Delta_{\text {eff }}=-2 \pi \times 30 \mathrm{kHz}$. The relative width of the central Bragg peak for the BEC is set to be $\mathcal{W}=1$. As the pump strength increases, the system transitions through all three phases, i.e., NP, SSF, and SMI. Panel (a) shows simulation results for $|\alpha|^{2}$ (blue squares) and $\mathcal{W}$ (red circles) of the steady states. Panel (b) shows experimental measurement of $N_{\mathrm{ph}}$ for the $T_{r}=40 \mathrm{~ms}$ ramping protocol (blue squares), as well as $N_{\mathrm{ph}}$ (blue crosses) and $\mathcal{W}$ (red circles) for the $T_{r}=20 \mathrm{~ms}$ ramping protocol. We note that a background radiation of $N_{\mathrm{ph}} \approx 2.7 \times 10^{4}$ is recorded as soon as the laser pump is switched on. The numbers indicate the representative points whose simulated and measured density distributions are shown in Fig. 4.

distributions in Fig. 4 . The numbering ( 1 to 6 ) of the quantum states in Fig. 4 refers to the different pump strengths indicated in Fig. 5(b).

As a BEC in the normal phase, the atomic density distribution form a Thomas-Fermi cloud in the real space [Fig. $4(\mathrm{~m})]$ and correspondingly a single blob in the momentum space [Fig. $4(a, g)]$. This can be observed both in experiments and simulations. The momentum space distribution has an elliptical shape in experiments but a circular shape in simulation. This is because the harmonic trap is anisotropic in the experimental setup $\omega_{x} \neq \omega_{y}$, while the confining potential in simulations [Eq. (12b)] is isotropic in the $x$ and $y$ directions.

In both experiments and simulations, the momentum space density distribution $\tilde{\rho}(\mathbf{k})$ in the SSF and SMI phases is completely different. It provides a way to track the phase boundary. A typical SSF state is represented by "State 2", whose measured and simulated momentum space densities are shown in Figs. 4(b) and 4(h), respectively. In the SSF phase, the central Bragg peak at $\left(p_{\mathrm{x}}, p_{\mathrm{y}}\right)=(0,0)$ is high and narrow and the satellite peaks are clearly visible. The four Bragg peaks at $\left(p_{\mathrm{x}}, p_{\mathrm{y}}\right)=\left( \pm \hbar k_{c}, \pm \hbar k_{c}\right)$ are the next dominant peaks and they indicate a strong coherence between atoms in the immediately neighboring sites of the checkerboard lattice, which are $\left( \pm \lambda_{c} / 2, \pm \lambda_{c} / 2\right)$ apart from each other. On top of these strong peaks, small peaks are seen at $\left(p_{\mathrm{x}}, p_{\mathrm{y}}\right)=\left(0, \pm 2 k_{c}\right)$ and 
barely visible at $\left(p_{\mathrm{x}}, p_{\mathrm{y}}\right)=\left( \pm 2 k_{c}, 0\right)$, which correspond to the optical pump lattice and the intracavity lattice, respectively. In contrast, "State 6" is a good representative of the SMI phase, whose measured and simulated momentum space densities are shown in Figs. 4(f) and $4(1)$, respectively. In the SMI phase, the central peak becomes broad and low, and the satellite peaks become diffuse. They indicate the strong localization of the atoms in the individual lattice sites and the lack of coherence between the atoms $15,18,22,24,52$ 56]. For comparison, the corresponding simulated real space density distributions $\rho(\mathbf{x})$ for the chosen states are also shown in Fig. $4(\mathrm{~m}-\mathrm{r})$. These images are not available from our experimental setup because the resolution of the absorption imaging system in the experiment is not good enough to resolve the individual lattice sites. The localization and loss of coherence of the atoms accompanying the increasing pump strength does not trigger qualitative change in the real space density distribution.

For different pump strengths at the fixed detuning $\Delta_{\text {eff }}=-2 \pi \times 30 \mathrm{kHz}$, we summarize the simulated cavity field magnitude $|\alpha|^{2}$, the measured intracavity photon number $N_{\mathrm{ph}}$, as well as the simulated and measured relative widths $\mathcal{W}$ of the central Bragg peak in Fig. 5. As further discussed in Appendix B, $N_{\text {ph }}$ clearly manifests the retardation effect for the NP-SSF transition for the faster ramping protocol. Meanwhile, the NP-SSF boundary measured from the slower protocol is close to the simulated steady-state boundary. There is roughly an order of magnitude difference between the experimentally measured $N_{\text {ph }}$ [Fig. 5(b)] and the simulated $|\alpha|^{2}$ [Fig. 5(a)], which is consistent with the scaling presented in Eq. (8). On the other hand, the relative width $\mathcal{W}$ is almost insensitive to the self-organization, but increases drastically as soon as Mott insulation kicks in. The relative width has seemingly a larger increase in experiments when compared to simulations. We attribute this to the initial BEC cloud that has different shapes in experiments and simulations due to the different confining potentials.

The boundary between the SSF and SMI phases is defined by the onset of the broadening of the central Bragg peak in the momentum distribution. This criterion is used both in simulation and experiment. More specifically, for the experimental data, we fit a line, shown as the red solid line in Fig. 5(b), using the first five data points after $\mathcal{W}$ starts to increase. The crossing with the initial width, i.e., the horizontal red dashed line, marks the SSF to SMI phase boundary. The obtained experimental and simulated SSF-SMI boundaries are shown in Fig. 2 as black and blue lines, respectively. The two boundaries agree well with each other at large negative detunings $\left|\Delta_{\text {eff }}\right| \geq 2 \pi \times 30 \mathrm{kHz}$, but deviate at small detunings. This discrepancy is mainly due to the dynamical retardation effects as discussed in Sec. 2.2 and Appendix B, whose simulation requires a prohibitatively large amount of computational resources. Nevertheless, our results show that the experimental and simulation results are generally in good agreement.

\section{Conclusions and Discussion}

We have used MCTDH-X to quantitatively determine the SSF-SMI boundary of a recoil resolved cavity-BEC system. This is the first time that MCTDH-X simulation results are directly compared quantitatively to experimental results for a cavity-BEC system, and the comparison is non-trivial due to limitation in computational resources. In contrast to the significant dynamical effects at play and a relatively large size of superlattice, our twodimensional simulations are limited to steady states and a small number of superlattice sites. These computational difficulties can be judiciously circumvented by choosing different ramping rates for the measurement of different quantities on the experimental side, as well as simplifying the full lattice to a minimal four-well representation in the simulation. 
The systematic errors of our proposed approach mainly stem from the small size of the lattice system used in simulations, and are small when expressed in terms of the pump rate. We have thereby established MCTDH-X a feasible numerical method for the quantitative calculation of the superfluid-Mott-insulator boundary in an ultracold atomic system which forms a superlattice with a large number of atoms per site.

It is also worthwhile to perform a comparison between our approach and other existing approaches for the purpose of determining the SSF-SMI boundary. The alternative approaches are usually based on the mapping to the Bose-Hubbard model. Given the effective optical lattice potential, the Wannier functions can be estimated by different numerical methods, many of which are available for quantum optical systems 69 72]. The Wannier functions then allow the extraction of the Bose-Hubbard parameters $t$ and $U$ [cf. Eq. (4)], which can be further used to determine the superfluid-Mott-insulator boundary. The last step can be performed by utilizing an empirical formula [58]. This approach no longer suffers from the finite size effect in comparison to our proposed simplification scheme. Nevertheless, when calculating the Wannier functions, single-particle wave functions are usually considered, and a broadening of Wannier function due to on-site interaction is generally not taken into account 73,74. This could result in an underestimation in the Bose-Hubbard ratio $t / U$, and give rise to a different kind of systematic errors in comparison to our proposed MCTDH-X scheme.

\section{Acknowledgements}

We acknowledge the computation time on the ETH Euler cluster and the Hawk cluster at the HLRS Stuttgart.

Funding information R.L. and R.Ch. acknowledge funding from the Swiss National Science Foundation (SNSF) and the ETH Grants; Ch.G., J.K., A.H. and H.K. acknowledge funding from the Deutsche Forschungsgemeinschaft through the SFB 925 and DFGKE2481/1-1; P.M. acknowledges funding from the ESPRC Grant no. EP/P009565/1; M.B. and A.U.J.L. acknowledge funding from the Austrian Science Foundation (FWF) under grant P32033.

\section{A Summary of methods and parameters}

The methods and parameters used in the experiments and simulations are summarized in Table 1 .

\section{B Experimental phase diagram with fast ramping protocol}

For a complementary comparison between the slow and fast ramping protocols in experiments, we show in Fig. 6 the phase diagram for the fast ramping protocol with $T_{r}=20 \mathrm{~ms}$. Compared to the steady-state phase diagram shown in Fig. 2 of the main text, the dynamical NP-SSF boundary for the fast protocol is indeed apparently shifted to higher pump strength due to retardation effect during the self-organization process. Importantly, at small detunings $\Delta_{\text {eff }}=-12.5 \mathrm{kHz}$ and $-17.5 \mathrm{kHz}$, the onset of the self-organization in experiments takes place later than the loss of superfluidity predicted by the simulations. As discussed in the main text, this accounts for the discrepancy between the simulated 
Table 1: Summary of the experimental and computational methods and parameters. Here $a_{B}$ is the Bohr radius.

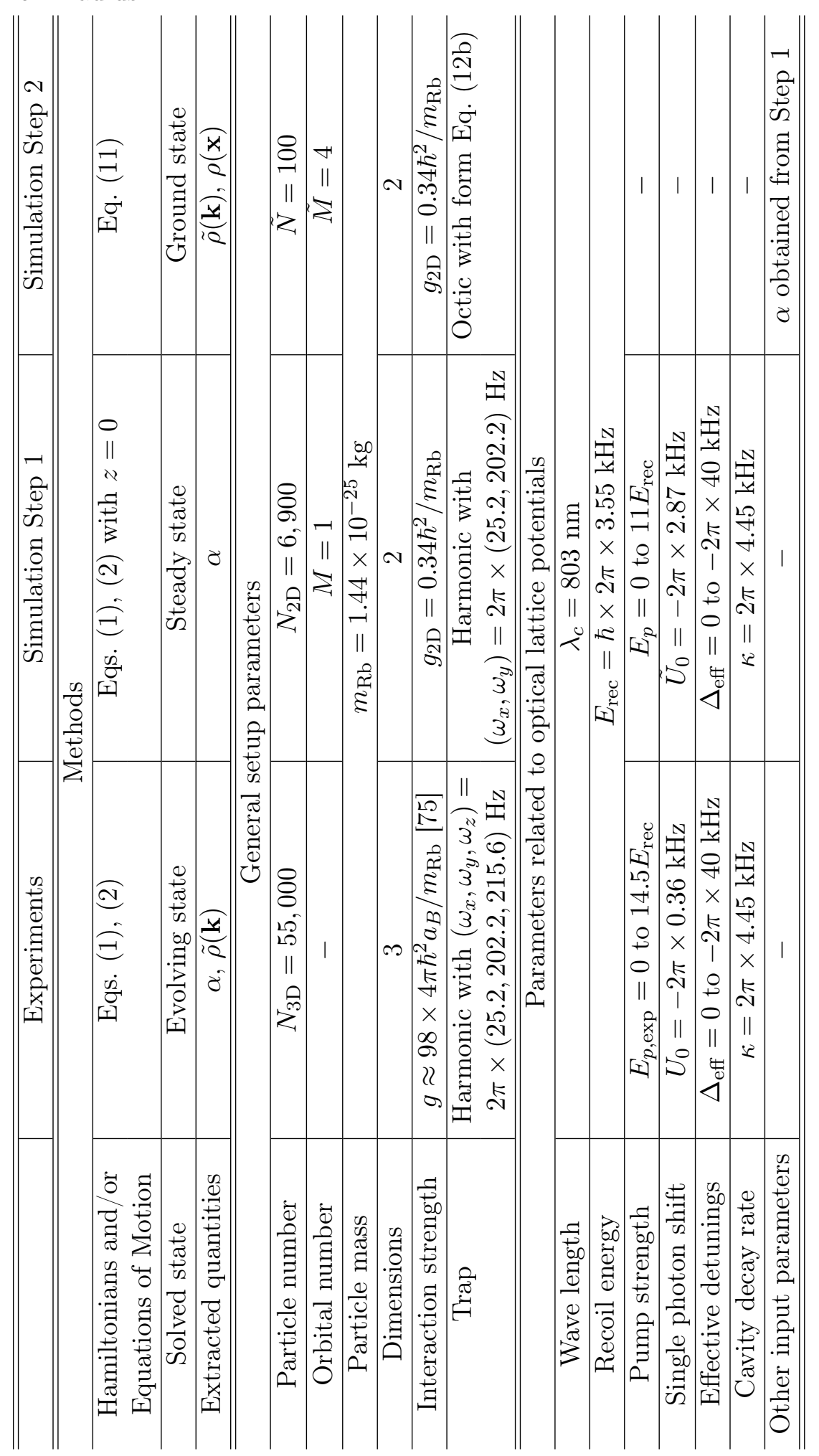

and experimental SSF-SMI boundaries. 


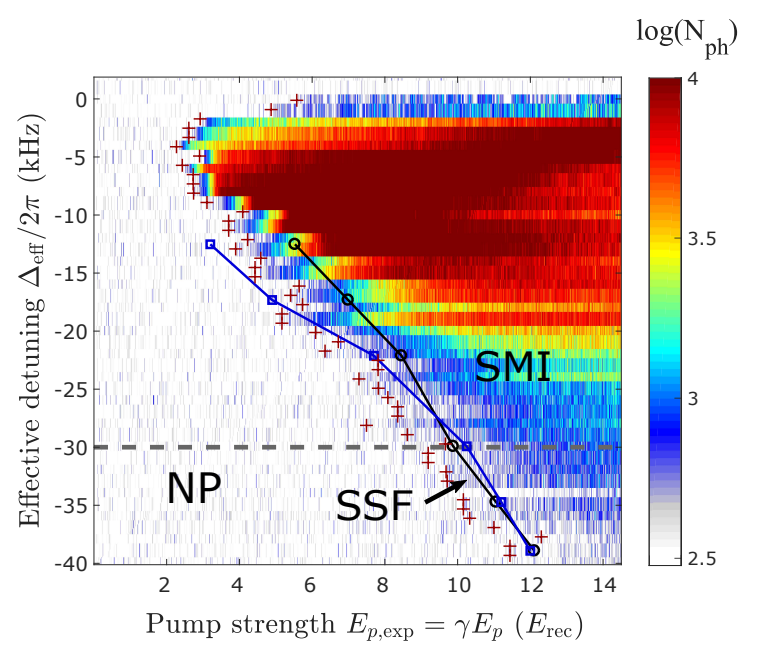

Figure 6: Experimental phase diagram for the fast ramping protocol with ramping time $T_{r}=20 \mathrm{~ms}$. The photon number is shown as color scale in the background, and the NPSSF boundary is shown as dark red crosses. The SSF-SMI boundary is shown as black circles. For comparison, the simulated SSF-SMI boundaries is shown by the blue squares. The experimental and simulation data for the SSF-SMI boundary are also used for the steady-state phase diagram in Fig. 2 of the main text.

\section{Multiconfigurational time-dependent Hartree method}

The Multiconfigurational Time-Dependent Hartree Method for Indistinguishable Particles [34 36, 38, 39] is implemented in the MCTDH-X software [37], and can accurately simulate cavity-BEC systems. We consider a general Hamiltonian containing a one-body potential $V(\mathbf{x})$ and a two-body interaction $W\left(\mathbf{x}, \mathbf{x}^{\prime}\right)$ :

$$
\hat{\mathcal{H}}=\int d \mathbf{x} \hat{\Psi}^{\dagger}(\mathbf{x})\left\{\frac{p^{2}}{2 m}+V(\mathbf{x})\right\} \hat{\Psi}(\mathbf{x})+\frac{1}{2} \int d \mathbf{x} \hat{\Psi}^{\dagger}(\mathbf{x}) \hat{\Psi}^{\dagger}\left(\mathbf{x}^{\prime}\right) W\left(\mathbf{x}, \mathbf{x}^{\prime}\right) \hat{\Psi}(\mathbf{x}) \hat{\Psi}\left(\mathbf{x}^{\prime}\right),
$$

With the MCTDH-X approach, the many-body wave function follows the ansatz

$$
|\Psi(t)\rangle=\sum_{\mathbf{n}} C_{\mathbf{n}}(t) \prod_{k=1}^{M}\left[\frac{\left(\hat{b}_{k}^{\dagger}(t)\right)^{n_{k}}}{\sqrt{n_{k} !}}\right]|\mathrm{vac}\rangle,
$$

where $N$ is the number of atoms, $M$ is the number of single-particle wave functions (orbitals) and $\mathbf{n}=\left(n_{1}, n_{2}, \ldots, n_{M}\right)$ gives the number of atoms in each orbital, i.e. $\sum_{k=1}^{M} n_{k}=$ $N$. The time-dependent operator $\hat{b}_{k}^{\dagger}$ creates one atom in the $i$-th orbital $\psi_{i}(x)$

$$
\hat{b}_{i}^{\dagger}(t)=\int \psi_{i}^{*}(\mathbf{x} ; t) \hat{\Psi}^{\dagger}(\mathbf{x} ; t) d x .
$$

The time-evolution of the coefficients $C_{\mathbf{n}}(t)$ and the orbitals $\psi_{i}(\mathbf{x} ; t)$ are obtained using the time-dependent variational principle 76$]$.

\section{Calculation of the effective two-dimensional atomic con- tact interaction strength}

The atomic contact interaction strength in the two-dimensional system is calculated based on the Thomas-Fermi approximation. By assuming a strong interaction and comparatively 
vanishing kinetic energy, the Gross-Pitaevskii equation for the Thomas-Fermi cloud can be written as

$$
E_{0} \phi(x, y)=V_{\text {trap }}(x, y) \phi(x, y)+N g_{2 \mathrm{D}}|\phi(x, y)|^{2} \phi(x, y),
$$

where $\phi(x, y)$ is the single-particle wave function, $E_{0}$ is the energy of the system to be determined, $N$ is the atom number, $V_{\text {trap }}=\frac{m}{2}\left(\omega_{x}^{2} x^{2}+\omega_{y}^{2} y^{2}\right)$ is the harmonic trap, $g_{2 \mathrm{D}}$ is the effective two-dimensional interaction strength which we want to calculate. The atomic density profile $\rho(x, y)=|\phi(x, y)|^{2}$ thus follows:

$$
\rho(x, y) \equiv|\phi(x, y)|^{2}=-\frac{m}{2 N g_{2 \mathrm{D}}}\left(\omega_{x}^{2} x^{2}+\omega_{y}^{2} y^{2}\right)+E_{0},
$$

and vanishes at $\rho\left(r_{x}, 0\right)=\rho\left(0, r_{y}\right)=0$, with the Thomas-Fermi radii

$$
\omega_{x} r_{x}=\omega_{y} r_{y}=\sqrt{\frac{2 E_{0} N g_{2 \mathrm{D}}}{m}} \equiv \tilde{r}_{0}
$$

The normalization of the density distribution requires $\int_{\Omega} d x d y \rho(x, y)=1$ where the integration region $\Omega=\{x, y \mid \rho(x, y)>0\}=\left\{x, y \mid \omega_{x}^{2} r_{x}^{2}+\omega_{y}^{2} y^{2}<\tilde{r}_{0}^{2}\right\}$ is an ellipse. This solves the system energy:

$$
E_{0}=\sqrt{\frac{m \omega_{x} \omega_{y}}{\pi N g}}
$$

Combining the equations above, the two-dimensional interaction strength is

$$
N g_{2 \mathrm{D}}=\frac{\pi m}{4} \frac{r_{x}^{4} \omega_{x}^{3}}{\omega_{y}}=\frac{\pi m}{4} \frac{r_{y}^{4} \omega_{y}^{3}}{\omega_{x}}
$$

\section{E Effects induced by the confining potential and the size of the reduced lattice}

In Sec. 3.3 we have argued that, for the purpose of determining the SSF-SMI boundary in terms of $E_{p}$, it is enough to use the unit cell with four sites to represent the full lattice. This is because size effect only slightly affects the transition point in terms of the Bose-Hubbard parameter ratio $t / U$, which in turn has an exponential dependence on the pump strength $E_{p}$. Furthermore, we have argued that the simulated boundary is almost insensitive to the confining potential $V_{\text {conf }}$, which we use to impose the boundary condition for the small lattice cell. In this Appendix we confirm these arguments by performing simulations with a different number of lattice sites and different confining potentials, and the results are summarized in Fig. 7 .

We reproduce the simulations in Sec. 3.3 with confining potentials different from the one presented in Eq. (12b). The confining potentials we use for Fig. 7 share the form

$$
V_{\text {conf }}(x, y)=E_{\text {conf }}\left(x^{2}+y^{2}\right)^{8} / \lambda_{c}^{16} .
$$

For a fixed effective detuning $\Delta_{\text {eff }}=-2 \pi \times 30 \mathrm{kHz}$ and varying pump strength $E_{p}$, we use different confining potential strengths $E_{\text {conf }}$ on top of the optical lattice $\tilde{V}_{\text {opt }}(x, y)$, and choose the configuration parity of the lattice according to our need. The following 

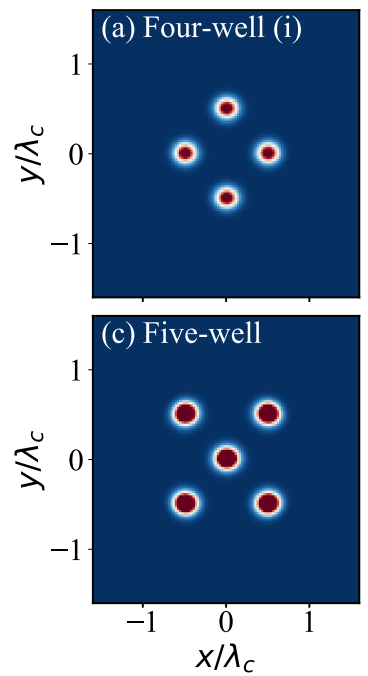
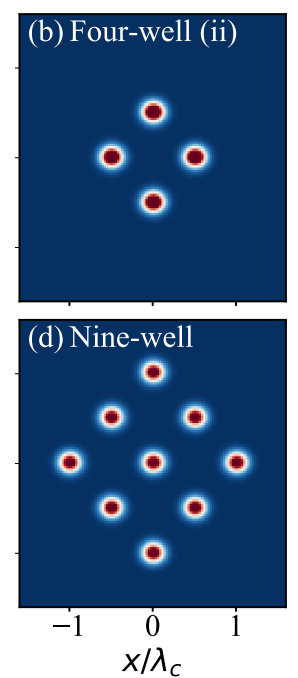

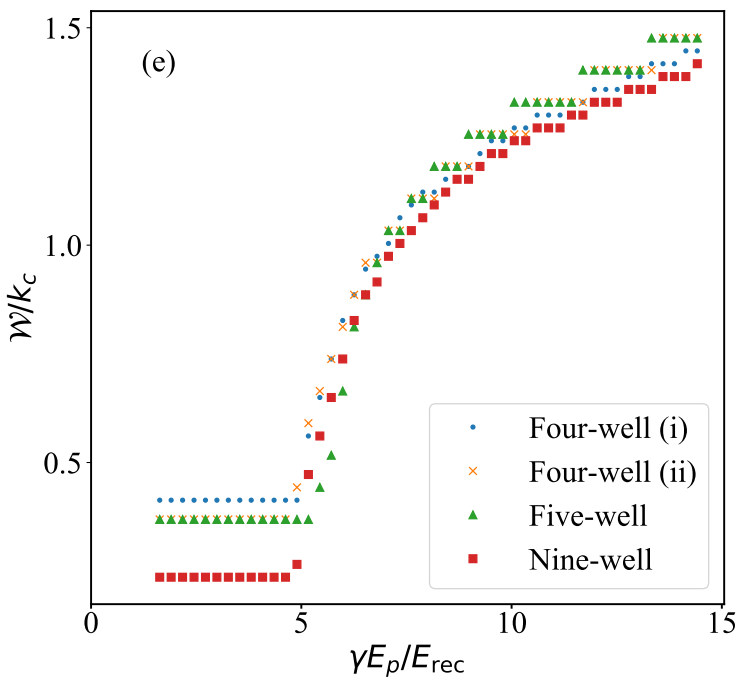

Figure 7: (a-d) Exemplary real-space density distributions of (a) the four-well system described by Eq. 12b in Sec. 3.3. (b) an alternative realization of the four-well system, (c) a five-well system, and (d) a nine-well system. (e) The width $\mathcal{W}$ of the central peak in the momentum space as a function of the pump strength $E_{p}$ in these four systems.

combinations of confining potential strengths and lattice configuration parities are chosen:

$$
\begin{array}{cl}
E_{\text {conf }, 4 \text { well(ii) }}=20 E_{\text {rec }}, & \text { odd lattice } \\
E_{\text {conf }, 5 \text { well }}=10 E_{\text {rec }}, & \text { even lattice } \\
E_{\text {conf }, 9 \text { well }}=0.01 E_{\text {rec }}, & \text { even lattice. }
\end{array}
$$

These combinations respectively produce an alternative realization of the four-well system, a five-well system, and a nine-well system. Their exemplary real-space density distributions are shown in Fig. 7(a-d).

In order to make the computational effort feasible and the simulation results comparable, we impose a filling factor of $\nu=1$ for all the four cases. As a result, the number of atoms $N$ and the number of orbitals $M$ are both equal to the number of wells in the simulations. We summarize the width $\mathcal{W}$ of the central peak in the momentum space for different confining potentials in Fig. 7)(e).

In the SSF phase, the width $\mathcal{W}$ is sensitive to the confining potential, and similar effects has been seen in Fig. 5 in the main text. This sensitivity contributes to a slight variance in the predicted SSF-SMI boundary for different confining potentials. Nevertheless, in all the four scenarios that we investigate in this Appendix, the SSF-SMI boundary is predicted to take place at roughly the same pump strength, with a variance of roughly $0.5 E_{\text {rec }}$. We can thus confirm that, for the determination of the SSF-SMI boundary in terms of the pump strength, a small system with four lattice sites is enough and the sensitivity on the form of the confining potential is small. We note that now the SSF-SMI transition takes place at a smaller pump strength than the results in Fig. 5(a) because of the low filling factor.

\section{References}

[1] I. Bloch, Ultracold quantum gases in optical lattices, Nature Physics 1, 23 (2005), doi:https://doi.org/10.1038/nphys138. 
[2] I. Bloch, J. Dalibard and S. Nascimbène, Quantum simulations with ultracold quantum gases, Nature Physics 8, 267 (2012), doi:https://doi.org/10.1038/nphys2259.

[3] C. Gross and I. Bloch, Quantum simulations with ultracold atoms in optical lattices, Science 357, 995 (2017), doi/https://doi.org/10.1126/science.aal3837.

[4] F. Mivehvar, F. Piazza, T. Donner and H. Ritsch, Cavity qed with quantum gases: New paradigms in many-body physics (2021), arXiv:2102.04473.

[5] H. Ritsch, P. Domokos, F. Brennecke and T. Esslinger, Cold atoms in cavitygenerated dynamical optical potentials, Reviews of Modern Physics 85, 553 (2013), doi https://doi.org/10.1103/RevModPhys.85.553.

[6] M. Aspelmeyer, T. Kippenberg and F. Marquardt, Cavity optomechanics, Reviews of Modern Physics 86, 1391 (2014), doi:https://doi.org/10.1103/RevModPhys.86.1391.

[7] K. Baumann, C. Guerlin, F. Brennecke and T. Esslinger, Dicke quantum phase transition with a superfluid gas in an optical cavity, Nature 464, 1301 (2010), doi:10.1038/nature09009

[8] K. Baumann, R. Mottl, F. Brennecke and T. Esslinger, Exploring symmetry breaking at the dicke quantum phase transition, Phys. Rev. Lett. 107, 140402 (2011), doi:10.1103/PhysRevLett.107.140402.

[9] J. Klinder, H. Keßler, M. Wolke, L. Mathey and A. Hemmerich, Dynamical phase transition in the open dicke model, Proceedings of the National Academy of Sciences 112(11), 3290 (2015), doi:10.1073/pnas.1417132112.

[10] R. Mottl, F. Brennecke, K. Baumann, R. Landig, T. Donner and T. Esslinger, Rotontype mode softening in a quantum gas with cavity-mediated long-range interactions, Science 336(6088), 1570 (2012), doi:10.1126/science.1220314.

[11] L. Hruby, N. Dogra, M. Landini, T. Donner and T. Esslinger, Metastability and avalanche dynamics in strongly correlated gases with long-range interactions, Proceedings of the National Academy of Sciences 115(13), 3279 (2018), doi: $10.1073 /$ pnas. 1720415115 .

[12] P. Molignini, L. Papariello, A. U. J. Lode and R. Chitra, Superlattice switching from parametric instabilities in a driven-dissipative bose-einstein condensate in a cavity, Phys. Rev. A 98, 053620 (2018), doi 10.1103/PhysRevA.98.053620.

[13] R. M. Kroeze, Y. Guo, V. D. Vaidya, J. Keeling and B. L. Lev, Spinor selfordering of a quantum gas in a cavity, Phys. Rev. Lett. 121, 163601 (2018), doi:10.1103/PhysRevLett.121.163601.

[14] H. Keßler, J. G. Cosme, C. Georges, L. Mathey and A. Hemmerich, From a continuous to a discrete time crystal in a dissipative atom-cavity system, New Journal of Physics 22(8), 085002 (2020), doi:10.1088/1367-2630/ab9fc0.

[15] J. Klinder, H. Keßler, M. R. Bakhtiari, M. Thorwart and A. Hemmerich, Observation of a superradiant mott insulator in the dicke-hubbard model, Phys. Rev. Lett. 115, 230403 (2015), doi:10.1103/PhysRevLett.115.230403.

[16] M. Greiner, O. Mandel, T. Esslinger, T. W. Hänsch and I. Bloch, Quantum phase transition from a superfluid to a Mott insulator in a gas of ultracold atoms, Nature 415, 39 (2002), doi $10.1038 / 415039 a$ 
[17] I. B. Spielman, W. D. Phillips and J. V. Porto, Mott-insulator transition in a two-dimensional atomic bose gas, Phys. Rev. Lett. 98, 080404 (2007), doi:10.1103/PhysRevLett.98.080404.

[18] Y. Kato, Q. Zhou, N. Kawashima and N. Trivedi, Sharp peaks in the momentum distribution of bosons in optical lattices in the normal state, Nature Phys. 4, 617 (2008), doi:10.1038/nphys983.

[19] R. Landig, L. Hruby, N. Dogra, M. Landini, R. Mottl, T. Donner and T. Esslinger, Quantum phases from competing short- and long-range interactions in an optical lattice, Nature 532, 476 (2016), doi:10.1038/nature17409.

[20] Y. Li, L. He and W. Hofstetter, Lattice-supersolid phase of strongly correlated bosons in an optical cavity, Phys. Rev. A 87, 051604 (2013), doi:10.1103/PhysRevA.87.051604.

[21] M. R. Bakhtiari, A. Hemmerich, H. Ritsch and M. Thorwart, Nonequilibrium phase transition of interacting bosons in an intra-cavity optical lattice, Phys. Rev. Lett. 114, 123601 (2015), doi:10.1103/PhysRevLett.114.123601.

[22] A. U. J. Lode and C. Bruder, Dynamics of hubbard hamiltonians with the multiconfigurational time-dependent hartree method for indistinguishable particles, Phys. Rev. A 94, 013616 (2016), doi:10.1103/PhysRevA.94.013616.

[23] R. Lin, L. Papariello, P. Molignini, R. Chitra and A. U. J. Lode, Superfluid-mottinsulator transition of ultracold superradiant bosons in a cavity, Phys. Rev. A 100, 013611 (2019), doi:10.1103/PhysRevA.100.013611.

[24] R. Lin, P. Molignini, A. U. J. Lode and R. Chitra, Pathway to chaos through hierarchical superfluidity in blue-detuned cavity-bec systems, Phys. Rev. A 101, 061602 (2020), doi:10.1103/PhysRevA.101.061602.

[25] E. P. L. van Nieuwenburg, Y.-H. Liu and S. D. Huber, Learning phase transitions by confusion, Nature Physics 13(5), 435 (2017), doi:10.1038/nphys4037.

[26] F. Schäfer and N. Lörch, Vector field divergence of predictive model output as indication of phase transitions, Phys. Rev. E 99, 062107 (2019), doi 10.1103 /PhysRevE.99.062107.

[27] J. Arnold, F. Schäfer, M. Žonda and A. U. J. Lode, Interpretable and unsupervised phase classification (2020), arXiv:2010.04730.

[28] F. D'Angelo and L. Böttcher, Learning the ising model with generative neural networks, Phys. Rev. Research 2, 023266 (2020), doi 10.1103/PhysRevResearch.2.023266.

[29] S. Ahmed, C. S. Muñoz, F. Nori and A. F. Kockum, Classification and reconstruction of optical quantum states with deep neural networks (2020), arXiv:2012.02185.

[30] B. S. Rem, N. Käming, M. Tarnowski, L. Asteria, N. Fläschner, C. Becker, K. Sengstock and C. Weitenberg, Identifying quantum phase transitions using artificial neural networks on experimental data, Nature Physics 15(9), 917 (2019), doi:10.1038/s41567019-0554-0.

[31] A. U. J. Lode, R. Lin, M. Büttner, L. Papariello, C. Lévêque, R. Chitra, M. C. Tsatsos, D. Jaksch and P. Molignini, Optimized observable readout from single-shot images of ultracold atoms via machine learning (2020), arXiv:2010.14510 
[32] A. Valenti, G. Jin, J. Léonard, S. D. Huber and E. Greplova, Scalable hamiltonian learning for large-scale out-of-equilibrium quantum dynamics (2021), arXiv: 2103.01240 .

[33] L. S. Cederbaum, A. I. Streltsov and O. E. Alon, Fragmented metastable states exist in an attractive bose-einstein condensate for atom numbers well above the critical number of the gross-pitaevskii theory, Phys. Rev. Lett. 100, 040402 (2008), doi $10.1103 /$ PhysRevLett.100.040402

[34] A. U. J. Lode, Multiconfigurational time-dependent hartree method for bosons with internal degrees of freedom: Theory and composite fragmentation of multicomponent bose-einstein condensates, Phys. Rev. A 93, 063601 (2016), doi $10.1103 /$ PhysRevA.93.063601.

[35] O. E. Alon, A. I. Streltsov and L. S. Cederbaum, Multiconfigurational time-dependent hartree method for bosons: Many-body dynamics of bosonic systems, Phys. Rev. A 77, 033613 (2008), doi:10.1103/PhysRevA.77.033613.

[36] E. Fasshauer and A. U. J. Lode, Multiconfigurational time-dependent hartree method for fermions: Implementation, exactness, and few-fermion tunneling to open space, Phys. Rev. A 93, 033635 (2016), doi:10.1103/PhysRevA.93.033635.

[37] A. U. J. Lode, M. C. Tsatsos, E. Fasshauer, R. Lin, L. Papariello, P. Molignini, C. Lévêque and S. E. Weiner, MCTDH-X: the time-dependent multiconfigurational hartree for indistinguishable particles software (2021).

[38] R. Lin, P. Molignini, L. Papariello, M. C. Tsatsos, C. Lévêque, S. E. Weiner, E. Fasshauer, R. Chitra and A. U. J. Lode, MCTDH-x: The multiconfigurational time-dependent hartree method for indistinguishable particles software, Quantum Science and Technology 5(2), 024004 (2020), doi:10.1088/2058-9565/ab788b.

[39] A. U. J. Lode, C. Lévêque, L. B. Madsen, A. I. Streltsov and O. E. Alon, Colloquium: Multiconfigurational time-dependent hartree approaches for indistinguishable particles, Rev. Mod. Phys. 92, 011001 (2020), doi:10.1103/RevModPhys.92.011001.

[40] J. Klinder, H. Keßler, C. Georges, J. Vargas and A. Hemmerich, Bose-einstein condensates in an optical cavity with sub-recoil bandwidth, Applied Physics B 122, 299 (2016).

[41] C. Maschler, I. B. Mekhov and H. Ritsch, Ultracold atoms in optical lattices generated by quantized lightÂ fields, The European Physical Journal D 46(3), 545 (2008), doi:10.1140/epjd/e2008-00016-4.

[42] D. Nagy, G. Szirmai and P. Domokos, Self-organization of a bose-einstein condensate in an optical cavity, The European Physical Journal D 48(1), 127 (2008), doi $10.1140 /$ epjd/e2008-00074-6.

[43] D. Nagy, G. Kónya, G. Szirmai and P. Domokos, Dicke-model phase transition in the quantum motion of a bose-einstein condensate in an optical cavity, Phys. Rev. Lett. 104, 130401 (2010), doi:10.1103/PhysRevLett.104.130401.

[44] D. Nagy, G. Szirmai and P. Domokos, Critical exponent of a quantum-noise-driven phase transition: The open-system dicke model, Phys. Rev. A 84, 043637 (2011), doi:10.1103/PhysRevA.84.043637. 
[45] F. Brennecke, R. Mottl, K. Baumann, R. Landig, T. Donner and T. Esslinger, Real-time observation of fluctuations at the driven-dissipative dicke phase transition, Proceedings of the National Academy of Sciences 110(29), 11763 (2013), doi $10.1073 /$ pnas. 1306993110.

[46] R. H. Dicke, Coherence in spontaneous radiation processes, Phys. Rev. 93, 99 (1954), doi:10.1103/PhysRev.93.99

[47] K. Hepp and E. H. Lieb, On the superradiant phase transition for molecules in a quantized radiation field: the dicke maser model, Annals of Physics 76(2), 360 (1973), doi:https://doi.org/10.1016/0003-4916(73)90039-0.

[48] Y. K. Wang and F. T. Hioe, Phase transition in the dicke model of superradiance, Phys. Rev. A 7, 831 (1973), doi:10.1103/PhysRevA.7.831.

[49] H. Carmichael, C. Gardiner and D. Walls, Higher order corrections to the dicke superradiant phase transition, Physics Letters A 46(1), 47 (1973), doi:https://doi.org/10.1016/0375-9601(73)90679-8.

[50] M. Łącki, B. Damski and J. Zakrzewski, Locating the quantum critical point of the bose-hubbard model through singularities of simple observables, Scientific Reports 6(1), 38340 (2016), doi:10.1038/srep38340.

[51] O. A. Prośniak, M. Łacki and B. Damski, Critical points of the three-dimensional bose-hubbard model from on-site atom number fluctuations, Scientific Reports 9(1), 8687 (2019), doi:10.1038/s41598-019-44825-9.

[52] I. B. Spielman, W. D. Phillips and J. V. Porto, Condensate fraction in a 2d bose gas measured across the mott-insulator transition, Phys. Rev. Lett. 100, 120402 (2008), doi $10.1103 /$ PhysRevLett.100.120402

[53] S. E. Weiner, M. C. Tsatsos, L. S. Cederbaum and A. U. J. Lode, Phantom vortices: hidden angular momentum in ultracold dilute bose-einstein condensates, Scientific Reports 7, 40122 EP (2017).

[54] P. B. Blakie and C. W. Clark, Wannier states and bose-hubbard parameters for $2 d$ optical lattices, Journal of Physics B: Atomic, Molecular and Optical Physics 37(7), 1391 (2004), doi: $10.1088 / 0953-4075 / 37 / 7 / 002$.

[55] B. Chatterjee and A. U. J. Lode, Order parameter and detection for a finite ensemble of crystallized one-dimensional dipolar bosons in optical lattices, Phys. Rev. A 98, 053624 (2018), doi:10.1103/PhysRevA.98.053624.

[56] B. Chatterjee, C. Lévêque, J. Schmiedmayer and A. U. J. Lode, Detecting onedimensional dipolar bosonic crystal orders via full distribution functions, Phys. Rev. Lett. 125, 093602 (2020), doi:10.1103/PhysRevLett.125.093602.

[57] M. P. A. Fisher, P. B. Weichman, G. Grinstein and D. S. Fisher, Boson localization and the superfluid-insulator transition, Phys. Rev. B 40, 546 (1989), doi: $10.1103 /$ PhysRevB.40.546.

[58] I. Danshita and A. Polkovnikov, Superfluid-to-mott-insulator transition in the onedimensional bose-hubbard model for arbitrary integer filling factors, Phys. Rev. A 84, 063637 (2011), doi:10.1103/PhysRevA.84.063637. 
[59] N. Teichmann, D. Hinrichs, M. Holthaus and A. Eckardt, Bose-hubbard phase diagram with arbitrary integer filling, Phys. Rev. B 79, 100503 (2009), doi:10.1103/PhysRevB.79.100503.

[60] K. V. Krutitsky, Ultracold bosons with short-range interaction in regular optical lattices, Physics Reports 607, 1 (2016), doi:https://doi.org/10.1016/j.physrep.2015.10.004, Ultracold bosons with shortrange interaction in regular optical lattices.

[61] S. Friebel, C. D'Andrea, J. Walz, M. Weitz and T. W. Hänsch, Co2laser optical lattice with cold rubidium atoms, Phys. Rev. A 57, R20 (1998), doi:https://doi.org/10.1103/PhysRevA.57.R20

[62] T. A. Savard, K. M. O'Hara and J. E. Thomas, Laser-noise-induced heating in far-off resonance optical traps, Phys. Rev. A 56, R10950 (1997), doi:https://doi.org/10.1103/PhysRevA.56.R1095.

[63] A. U. J. Lode, K. Sakmann, O. E. Alon, L. S. Cederbaum and A. I. Streltsov, Numerically exact quantum dynamics of bosons with time-dependent interactions of harmonic type, Phys. Rev. A 86, 063606 (2012), doi:10.1103/PhysRevA.86.063606.

[64] K. Sakmann, A. I. Streltsov, O. E. Alon and L. S. Cederbaum, Quantum dynamics of attractive versus repulsive bosonic josephson junctions: Bosehubbard and full-hamiltonian results, Phys. Rev. A 82, 013620 (2010), doi $10.1103 /$ PhysRevA.82.013620.

[65] K. Sakmann, A. I. Streltsov, O. E. Alon and L. S. Cederbaum, Time-dependent restricted-active-space self-consistent-field theory for bosonic many-body systems, New Journal of Physics 13, 043003 (2011), doi:10.1088/1367-2630/13/4/043003.

[66] A. U. J. Lode, F. S. Diorico, R. Wu, P. Molignini, L. Papariello, R. Lin, C. Lévêque, L. Exl, M. C. Tsatsos, R. Chitra and N. J. Mauser, Many-body physics in twocomponent bose-einstein condensates in a cavity: fragmented superradiance and polarization, New Journal of Physics 20(5), 055006 (2018).

[67] C. Lévêque and L. B. Madsen, Time-dependent restricted-active-space self-consistentfield theory for bosonic many-body systems, New Journal of Physics 19(4), 043007 (2017), doi:10.1088/1367-2630/aa6319.

[68] N. March, The thomas-fermi approximation in quantum mechanics, Advances in Physics 6(21), 1 (1957), doi:10.1080/00018735700101156.

[69] N. Marzari and D. Vanderbilt, Maximally localized generalized wannier functions for composite energy bands, Phys. Rev. B 56, 12847 (1997), doi: $10.1103 /$ PhysRevB.56.12847.

[70] B. Wu, Y. Xu, L. Dong and J.-R. Shi, Self-consistent approach for mapping interacting systems in continuous space to lattice models, Chinese Physics Letters 29(8), 083701 (2012), doi:10.1088/0256-307x/29/8/083701.

[71] R. Walters, G. Cotugno, T. H. Johnson, S. R. Clark and D. Jaksch, Ab initio derivation of hubbard models for cold atoms in optical lattices, Phys. Rev. A 87, 043613 (2013), doi $10.1103 /$ PhysRevA.87.043613.

[72] S. Paul and E. Tiesinga, Wannier functions using a discrete variable representation for optical lattices, Phys. Rev. A 94, 033606 (2016), doi:10.1103/PhysRevA.94.033606 
[73] S. Zhu and B. Wu, Interaction effects on wannier functions for bosons in an optical lattice, Phys. Rev. A 92, 063637 (2015), doi:10.1103/PhysRevA.92.063637.

[74] M. Kremer, R. Sachdeva, A. Benseny and T. Busch, Interaction-induced effects on bose-hubbard parameters, Phys. Rev. A 96, 063611 (2017), doi $10.1103 /$ PhysRevA.96.063611.

[75] M. Egorov, B. Opanchuk, P. Drummond, B. V. Hall, P. Hannaford and A. I. Sidorov, Measurement of s-wave scattering lengths in a two-component bose-einstein condensate, Phys. Rev. A 87, 053614 (2013), doi:10.1103/PhysRevA.87.053614.

[76] P. Kramer and M. Saraceno, Geometry of the Time-Dependent Variational Principle in Quantum Mechanics, vol. 140 of Lecture Notes in Physics, Springer (1981). 